\title{
Production of multiply heavy flavoured baryons from Quark Gluon Plasma in relativistic heavy ion collisions
}

\author{
F. Becattini \\ Università di Firenze and \\ INFN Sezione di Firenze, Florence, Italy
}

\begin{abstract}
It is argued that in heavy ion collisions at LHC there could be a sizeable production of baryons containing two or three heavy quarks from statistical coalescence. This production mechanism is peculiar of Quark Gluon Plasma and the predicted rates, in heavy ion collisions at LHC energy, exceed those from a purely hadronic scenario, particularly for $\Xi_{b c}$ and $\Omega_{c c c}$. Thus, besides the interest in the discovery of these new states, enhanced ratios of these baryons over singly heavy flavoured hadrons, like B or D, in heavy ion collisions with respect to pp at the same energy, would be a clear indication of kinetic equilibration of heavy quarks in the Quark Gluon Plasma.
\end{abstract}

Charm and bottom quarks are expected to be abundantly produced in hadronic collisions at very high energies. In heavy ion collisions (HIC), multiple pair production is expected to occur, with average multiplicities which, at the LHC energy of $5.5 \mathrm{TeV}$, attain $\mathcal{O}(10)$ for bottom and $\mathcal{O}(100)$ for charm in central collisions. These quarks, produced in the early stage of the collision off hard scatterings, lose energy in the Quark Gluon Plasma (QGP) and, if the lifetime of the source is long enough, may reach thermal [1] (not chemical, as their annihilation rate is very low) equilibrium within the medium. At the hadronization point, they will coalesce into hadrons. If coalescence process occurs statistically at the hadronization temperature, there is a finite chance that two or even three of them coalesce into the same particle, thus giving rise to multiply heavy flavoured hadrons, particularly baryons. This phenomenon is likely to occur only if heavy quarks get very close to thermal equilibrium reshuffling over a large region because high momentum quarks, most likely, will hadronize into different particles unless two or three of them emerge very close in momentum from the hard process. The latter production mechanism, where multiply heavy flavoured hadrons arise from correlated quarks, predicts multiplicities which, at some large energy, are exceeded by those predicted by coalescence of uncorrelated quarks. The ultimate reason of this effect is that the average multiplicity of heavy quark pairs increases faster than soft hadrons multiplicity as a function of centre-of-mass energy. This is in turn related to the volume of the system at freeze-out. Consequently, the system gets denser in heavy quarks at chemical freeze-out as energy increases and so does the chance of formation of multiply heavy flavoured hadrons. Therefore, an enhanced production rate of these objects relative to singly heavy flavoured hadrons (like B's or D's) is distinctive of HIC and can be used as a probe of thermalization of heavy quarks within the QGP, thence as a signal of QGP itself. This idea was advocated in refs. 2, 3] where the authors envisaged an enhancement of $\mathrm{B}_{c}$ and $J / \psi$ mesons production from the QGP with respect to hadronic collisions at RHIC. In this paper, we amend and reinforce this picture by observing that the main advantage of baryons (and especially $\Xi_{b c}$ and $\Omega_{c c c}$ whose signal should be de- tectable at $\mathrm{LHC}$ ) over quarkonia and $\mathrm{B}_{c}$ is to enhance the difference from the "background" of coherent production, i.e. direct production of these states from early hard scatterings, possibly followed by melting in the plasma. Furthermore, in view of this relatively large production, HIC become a suitable place to discover many of these yet unobserved states.

That the production of multiply charmed baryons could be enhanced in high energy HIC was first proposed in ref. [4] on the basis of a quark recombination model. A different coalescence picture was used in ref. [5]. However, in both studies, no relation was set between production enhancement and heavy quark kinetic equilibration in the plasma and quantitative predictions of production rates were dependent on an undetermined free parameter. On the contrary, predictions are definite within the statistical coalescence model (SCM), whose basic idea has been introduced in ref. [3] following a work on statistical production of $J / \psi[\underline{6}]$, and used thereafter by many authors [7]. In practice, the SCM is the statistical hadronization model supplemented with the constraint of a fixed number of heavy quarks and antiquarks.

We are now going to calculate of the mean multiplicities $\left\langle n_{j}\right\rangle$ of heavy flavoured particles in the framework of the SCM. In HIC at high energy, electric charge, strangeness and baryon number conservation can be treated grand-canonically whereas charm and beauty conservation cannot because the multiplicities of heavy flavoured hadrons are not large. Thus, besides the number $N_{c}$ of $\mathrm{c}+\overline{\mathrm{c}}$ quarks and $N_{b}$ of $\mathrm{b}+\overline{\mathrm{b}}$ quarks, also the net charm $C$ and net beauty $B$ should be fixed. In fact, instead of $C, B, N_{c}, N_{b}$, any combination of these integers can be used to constrain the partition function. Indeed, it is advantegeous to use the numbers $\nu_{c}$ of $\mathrm{c}, \nu_{\bar{c}}$ of $\overline{\mathrm{c}}, \nu_{b}$ of $\mathrm{b}$ and $\nu_{\bar{b}}$ of $\overline{\mathrm{b}}$ quarks. The relevant partition function reads:

$$
\begin{aligned}
& Z\left(\nu_{c}, \nu_{\bar{c}}, \nu_{b}, \nu_{\bar{b}}\right)=Z_{l}\left[\prod_{f=c, \bar{c}, b, \bar{b}} \int_{-\pi}^{\pi} \frac{\mathrm{d} \phi_{f}}{2 \pi} \mathrm{e}^{1 \nu_{f} \phi_{f}}\right] \\
& \times \exp \left[\sum_{j} z_{j} \lambda_{j} \mathrm{e}^{-1 \nu_{c j} \phi_{c}-1 \nu_{\bar{c} j} \bar{\phi}_{c}-1 \nu_{b j} \phi_{b}-1 \nu_{\bar{b} j} \bar{\phi}_{b}}\right]
\end{aligned}
$$


where $Z_{l}$ is the grand-canonical partition function including all light-flavoured species; $z_{j}$ are the one-particle partition functions:

$$
z_{j}=\frac{g_{j} V}{2 \pi^{2}} m^{2} T \mathrm{~K}_{2}\left(\frac{m}{T}\right) \underset{m \gg T}{\simeq} g_{j} V\left(\frac{m T}{2 \pi}\right)^{3 / 2} \mathrm{e}^{-m / T} ;
$$

$\lambda_{j}$ are the fugacities (with regard to electric, baryonic and strangeness charge) and $\nu_{c j}, \nu_{\bar{c} j}, \nu_{b j}, \nu_{\bar{b} j}$ are the number of $\mathrm{c}, \overline{\mathrm{c}}, \mathrm{b}, \overline{\mathrm{b}}$ quarks respectively of the of the $j^{\text {th }}$ hadronic species; the factor $g_{j}$ in Eq. (2) is its spin degeneracy and $T$ is the temperature. The sum over $j$ in Eq. (10 involves all hadrons containing heavy quarks. The primary average multiplicity of any of these, in events with fixed numbers of heavy quarks, reads:

$$
\left\langle n_{j}\right\rangle=z_{j} \lambda_{j} \frac{Z\left(\nu_{c}-\nu_{c j}, \nu_{\bar{c}}-\nu_{\bar{c} j}, \nu_{b}-\nu_{b j}, \nu_{\bar{b}}-\nu_{\bar{b} j}\right)}{Z\left(\nu_{c}, \nu_{\bar{c}}, \nu_{b}, \nu_{\bar{b}}\right)}
$$

It can be realized that we would deal with factorized charm and bottom integrals in Eq. (II) were not for the presence of hadrons carrying both flavours, such as $\mathrm{B}_{c}$. To recover factorization, we can expand the integrand in Eq. (1) in power series of the $z_{j}$ of hadrons containing both c and b (anti-)quarks up to the first order. Defining:

$$
\begin{aligned}
\hat{Z}_{f}\left(\nu_{f}, \nu_{\bar{f}}\right)= & \int_{-\pi}^{\pi} \frac{\mathrm{d} \phi_{f}}{2 \pi} \frac{\mathrm{d} \bar{\phi}_{\bar{f}}}{2 \pi} \mathrm{e}^{1 \nu_{f} \phi_{f}+1 \nu_{\bar{f}} \bar{\phi}_{\bar{f}}} \\
& \times \exp \left[\sum_{j_{f}} z_{j_{f}} \lambda_{j_{f}} \mathrm{e}^{-1 \nu_{f j} \phi_{f}-1 \nu_{\bar{f} j} \bar{\phi}_{\bar{f}}}\right]
\end{aligned}
$$

where $f=c$ or $b$ and $j_{f}$ running over all hadrons containing $f$ or $\bar{f}$ quark, the partition function can be rewritten as:

$$
\begin{aligned}
& Z\left(\nu_{c}, \nu_{\bar{c}}, \nu_{b}, \nu_{\bar{b}}\right) \simeq Z_{l}\left[\hat{Z}_{c}\left(\nu_{c}, \nu_{\bar{c}}\right) \hat{Z}_{b}\left(\nu_{b}, \nu_{\bar{b}}\right)+\sum_{j_{c b}} z_{j_{c b}} \lambda_{j_{c b}}\right. \\
& \left.\times \hat{Z}_{c}\left(\nu_{c}-\nu_{c j}, \nu_{\bar{c}}-\nu_{\bar{c} j}\right) \hat{Z}_{b}\left(\nu_{b}-\nu_{b j}, \nu_{\bar{b}}-\nu_{\bar{b} j}\right)\right]
\end{aligned}
$$

where $j_{c b}$ runs over all hadrons containing both flavours. It is now clear from (5) that we will henceforth be dealing with factorized canonical partition functions because both $\hat{Z}_{c}$ and $\hat{Z}_{b}$ do no longer involve hadrons with both flavours. Whether stopping the expansion in (15) at first order is sufficient, will be discussed later. If we now denote by $a_{f n}$ the sum of $z_{j} \lambda_{j}$ for hadrons with $n$ units of open flavour $f, a_{\bar{f} n}$ for those with $n$ units of open antiflavour $\bar{f}$, and $a_{f 0}$ for $f \bar{f}$ states, we can calculate the $\hat{Z}_{f}$ in (4) by expanding in powers of $a_{f 0}$ :

$$
\begin{aligned}
& \hat{Z}_{f}\left(\nu_{f}, \nu_{\bar{f}}\right)=\int_{-\pi}^{\pi} \frac{\mathrm{d} \phi_{f}}{2 \pi} \frac{\mathrm{d} \bar{\phi}_{\bar{f}}}{2 \pi} \mathrm{e}^{1 \nu_{f} \phi_{f}+1 \nu_{\bar{f}} \bar{\phi}_{\bar{f}}} \\
& \times \exp \left[a_{f 0} \mathrm{e}^{-1 \phi_{f}-1 \bar{\phi}_{\bar{f}}}+\sum_{n=1}^{3} a_{f n} \mathrm{e}^{-1 n \phi_{f}}+a_{\bar{f} n} \mathrm{e}^{-1 n \bar{\phi}_{\bar{f}}}\right] \\
& =\sum_{h=0}^{\min \left(\nu_{f}, \nu_{\bar{f}}\right)} \frac{a_{f 0}^{h}}{h !} \zeta\left(\nu_{f}-h\right) \bar{\zeta}\left(\nu_{\bar{f}}-h\right)
\end{aligned}
$$

where:

$$
\zeta\left(\nu_{f}\right) \equiv \int_{-\pi}^{\pi} \frac{\mathrm{d} \phi_{f}}{2 \pi} \mathrm{e}^{1 \nu_{f} \phi_{f}} \exp \left[\sum_{n=1}^{3} a_{f n} \mathrm{e}^{-1 n \phi_{f}}\right]
$$

and similarly for $\bar{\zeta}\left(\nu_{\bar{f}}\right)$ with $a_{\bar{f} n}$ replacing the $a_{f n}$ 's. We note in passing that $\zeta\left(\nu_{f}\right)=0$ if $\nu_{f}<0$ and $\zeta(0)=1$. The mass hyerarchy in heavy flavoured hadrons make $a_{f 1} \gg a_{f 2} \gg a_{f 3}(f=c, b)$, because, according to (2), each term is suppressed with respect to the preceding one by a factor $\sim \exp \left(-m_{f} / T\right)$ where $m_{f}$ is the $\mathrm{c}$ or $\mathrm{b}$ quark mass. Neglecting $a_{f 3}$ (i.e. $\Omega_{c c c}$ or $\Omega_{b b b}$ baryons), the integrals in (17) give rise to a polynomial expression:

$$
\zeta\left(\nu_{f}\right) \simeq \sum_{k=0}^{\left[\nu_{f} / 2\right]} \frac{a_{f 1}^{\nu_{f}-2 k} a_{f 2}^{k}}{\left(\nu_{f}-2 k\right) ! k !}
$$

and likewise for $\bar{\zeta}$. The ratio between the $k^{\text {th }}$ and $(k-1)^{\text {th }}$ term in the above polynomial is less than $\left(a_{f 2} / a_{f 1}^{2}\right) \nu_{f}\left(\nu_{f}-1\right) / k \equiv R_{f} / k$. Recalling the definition of the $a_{f n}$ 's, one can roughly estimate it by assuming the mass of the hadrons to be the sum of the constituent masses of valence quarks and using Eq. (2):

$$
R_{f} \equiv \frac{a_{f 2}}{a_{f 1}^{2}} \nu_{f}\left(\nu_{f}-1\right) \approx \frac{\nu_{f}\left(\nu_{f}-1\right) \mathrm{e}^{2 m_{u, d} / T}}{g_{\mathrm{eff}} V\left[\frac{\left(m_{f}+m_{u}\right)^{2} T}{2 \pi m_{f}}\right]^{3 / 2}}
$$

where $g_{\text {eff }}(T, \lambda)$ is an effective degeneracy parameter including the spin degeneracy and the different states with the same numbers of heavy quarks, weighted by the ratio $z_{j} \lambda_{j} / z_{1} \lambda_{1}, z_{1}$ being the one-particle partition function of the lowest lying state. In Eq. (9) we tacitly assumed that $g_{\text {eff }}$ is the same for hadrons with one or two heavy quarks, which approximately holds according to our numerical check. From known states with one heavy quark, either c or b, one expects $g_{\text {eff }} \sim 10$ at $T=165 \mathrm{MeV}$ and $\lambda_{j}=1$. Taking constituent quark masses $m_{c}=1.54 \mathrm{GeV}, m_{b}=4.95 \mathrm{GeV}, m_{u, d}=0.33$ $\mathrm{GeV} m_{s}=0.51 \mathrm{GeV}$ and $T=165 \mathrm{MeV}$, i.e. the fitted chemical freeze-out temperature at very large energy [8, 9], it turns out that $R_{c} \sim \nu_{c}\left(\nu_{c}-1\right) / 0.34 \mathrm{fm}^{-3} V$ and $R_{b} \sim \nu_{b}\left(\nu_{b}-1\right) / 1.4 \mathrm{fm}^{-3} V$. Therefore, with the large volumes involved in HIC, $R_{c}, R_{b}$ are likely to be $\ll 1$ unless $\nu_{c}$ or $\nu_{b}$ are consistently large. In this case we can approximate the $\zeta$ in Eq. (8) with its first term, i.e. $\zeta\left(\nu_{f}\right) \simeq a_{f 1}^{\nu_{f}} / \nu_{f}$ !, so Eq. [6] becomes:

$$
\hat{Z}_{f}\left(\nu_{f}, \nu_{\bar{f}}\right) \simeq \sum_{h=0}^{\nu_{f}} \frac{a_{f 0}^{h}}{h !} \frac{a_{f 1}^{\nu_{f}-h}}{\left(\nu_{f}-h\right) !} \frac{a_{\bar{f} 1}^{\nu_{\bar{f}}-h}}{\left(\nu_{\bar{f}}-h\right) !}
$$

Again, the ratio between the $h^{\text {th }}$ and the $(h-1)^{\text {th }}$ term is less than $a_{f 0} \nu_{f} \nu_{\bar{f}} / a_{f 1} a_{\bar{f} 1} h$, which is approximately equal to $R_{f} / h$, with $R_{f}$ quoted in Eq. (9), because $\nu_{f}$ differs from $\nu_{\bar{f}}$ by few units and being $a_{f 1}$ very close to $a_{\bar{f} 1}$ if chemical potentials are not too large. Therefore, under the same conditions needed for its validity, the 
sum in (10) can be approximated with its first term, i.e. $\hat{Z}_{f}\left(\nu_{f}, \nu_{\bar{f}}\right) \simeq a_{f 1}^{\nu_{f}} a_{\bar{f} 1}^{\nu_{\bar{f}}} / \nu_{f} ! \nu_{\bar{f}} !$. By using this approximation, we can finally estimate the ratio between the first and the zeroth order terms in the expansion (5). For instance, for $\mathrm{B}_{c}$ mesons carrying one $\mathrm{c}$ and one $\mathrm{b}$ quark:

$$
\begin{aligned}
& \sum_{j_{c b}} z_{j_{c b}} \lambda_{j_{c b}} \frac{\hat{Z}_{c}\left(\nu_{c}-1, \nu_{\bar{c}}\right) \hat{Z}_{b}\left(\nu_{b}, \nu_{\bar{b}}-1\right)}{\hat{Z}_{c}\left(\nu_{c}, \nu_{\bar{c}}\right) \hat{Z}_{b}\left(\nu_{b}, \nu_{\bar{b}}\right)} \\
\simeq & \sum_{j_{c b}} z_{j_{c b}} \frac{\nu_{c} \nu_{\bar{b}}}{a_{c 1} a_{\bar{b} 1}} \approx \frac{\nu_{c} \nu_{\bar{b}} \mathrm{e}^{2 m_{u, d} / T}}{g_{\mathrm{eff}} V\left[\frac{m_{c} m_{b} T}{2 \pi\left(m_{c}+m_{b}\right)}\right]^{3 / 2}} \equiv R_{c b}(
\end{aligned}
$$

For hadrons with two b's and one c and viceversa, it can be easily shown that this ratio is even smaller. If $R_{c b} \ll 1$ (which is likely to be), the first order term in the expansion of the partition function (5) is negligible. Under these circumstances, and provided that the aforementioned conditions on $R_{c}, R_{b}$ are met, the primary average multiplicity of heavy flavoured hadrons for fixed number of $\mathrm{c}, \overline{\mathrm{c}}, \mathrm{b}, \overline{\mathrm{b}}$ quarks is especially simple:

$$
\left\langle n_{j}\right\rangle=z_{j} \lambda_{j} \prod_{f=c, \bar{c}, b, \bar{b}, \nu_{f j}>0} \frac{\nu_{f}\left(\nu_{f}-1\right) \ldots\left(\nu_{f}-\nu_{f j}+1\right)}{a_{f 1}^{\nu_{f j}}}
$$

The (12) is to be further averaged over the multiplicity distribution $p_{\nu_{c}}$ of $c \bar{c}$ and $p_{\nu_{b}}$ of b $\overline{\mathrm{b}}$ pairs created in a single collision. If they are independently produced, this is a Poisson distribution and, for open flavoured hadrons, the sum (12) yields its factorial moments, i.e.:

$$
\left\langle\left\langle n_{j}\right\rangle\right\rangle=z_{j} \lambda_{j} \prod_{f=c, \bar{c}, b, \bar{b}}\left(\frac{\left\langle\nu_{f}\right\rangle}{a_{f 1}}\right)^{\nu_{f j}} \equiv z_{j} \lambda_{j} \prod_{f=c, \bar{c}, b, \bar{b}} \eta_{f}^{\nu_{f j}}
$$

whereas for quarkonia it is more complicated since $\nu_{c}=$ $\nu_{\bar{c}}$ and $\nu_{b}=\nu_{\bar{b}}$. Eq. (13) is our final formula. As has been mentioned, it is an approximated expression valid if $R_{c}, R_{b}, R_{c b} \ll 1$. However, it can be shown, by performing an asymptotic expansion of the integral (61) [10] that it still holds under the weaker condition $R_{f} / \nu_{f} \ll 1$ if $\nu_{f}$ is large. Altogether, the Eq. (13) implies that the contribution of hadrons carrying more than one heavy flavoured quark in the balance equations $\sum_{j}\left\langle n_{j}\right\rangle \nu_{f j}=\left\langle\nu_{f}\right\rangle$ is neglected. It is interesting to note that the enhancement factors $\eta_{f}=\left\langle\nu_{f}\right\rangle / a_{f 1}$ are proportional to the density of heavy quarks at the hadronization temperature, as $a_{f 1} \propto V$. Therefore, the ratio between multiply and singly heavy flavoured hadrons, proportional to $\left(\left\langle\nu_{f}\right\rangle / V\right)^{\nu_{f j}-1}$, increases with centre-of-mass energy because the volume (or the charged multiplicity) increases much slower than $\sigma_{c \bar{c}}$ and $\sigma_{b \bar{b}}$ do.

The formula (13) can now be applied to estimate the average multiplicity of multiply heavy flavoured hadrons in HIC at RHIC and LHC. For sake of simplicity, we will confine ourselves to full phase space integrated quantities, disregarding spectra. To get started, we need the cross sections $\sigma_{c \bar{c}}$ and $\sigma_{b \bar{b}}$ in pp collisions at relevant energies. There is a large uncertainty on these values; recent calculations indicate $\sigma_{c \bar{c}}=110-656 \mu \mathrm{b}$ and $\sigma_{b \bar{b}}=1.2-2.86 \mu \mathrm{b}$ at $\sqrt{s}=200 \mathrm{GeV}$ [1] and $\sigma_{c \bar{c}}=3.4-9.2 \mathrm{mb}$ and $\sigma_{b \bar{b}}=88-260 \mu \mathrm{b}$ at $\sqrt{s}=5.5 \mathrm{TeV}$ [12]. The production of heavy quark pairs is a hard process and should scale like the number of collisions $N_{\text {coll }}$ in the Glauber model. Specifically, if $\sigma_{\text {inel }}$ is the total inelastic NN cross section, the average multiplicity of $c \overline{\mathrm{c}}$ pairs is $\left\langle\nu_{f}\right\rangle=\left\langle\nu_{\bar{f}}\right\rangle=N_{\text {coll }} \sigma_{f \bar{f}} / \sigma_{\text {inel }}$. At RHIC, in Au-Au collisions at $\sqrt{s}_{N N}=200 \mathrm{GeV}, \sigma_{\text {inel }} \simeq 42 \mathrm{mb}$ and for a $5.5 \%$ centrality selected sample, corresponding to an impact parameter range 0-3.5 fm, $N_{\text {coll }}=1080$ [13]. Thus, the average multiplicity of $c \overline{\mathrm{c}}$ pairs ranges from 2.8 to 17 , whereas for $\mathrm{b} \bar{b}$ pairs from 0.03 to 0.07 . On the other hand, at LHC, in $\mathrm{Pb}-\mathrm{Pb}$ collisions at $\sqrt{s}_{N N}=5.5 \mathrm{TeV}$, $\sigma_{\text {inel }} \simeq 60 \mathrm{mb}$ and for a $5.1 \%$ centrality selected sample, corresponding to the same impact parameter above, $N_{\text {coll }}=1670$ 13]. In this latter case, the average multiplicity of cc pairs ranges from 95 to 256 and from 2.4 to 7.2 for b $\bar{b}$ pairs. It should be noted that these estimates do not take into account possible structure function saturation effects, which are predicted to reduce heavy quark cross section at LHC [14]. Since there is not a clearcut evidence of this phenomenon as yet, we stick to the traditional picture of $N_{\text {coll }}$ scaling, though this possibility is worthy of consideration in the future.

The other key ingredient in our calculation is the volume $V$. In order to extrapolate from SPS to RHIC, we take advantage of the fact that $V$ is proportional to the average multiplicity of pions. In $\mathrm{Pb}-\mathrm{Pb}$ at SPS at $\sqrt{s}_{N N}=17.2 \mathrm{GeV}, V \simeq 3.510^{3} \mathrm{fm}^{3}$ in full phase space [9], and $\left\langle\pi^{+}+\pi^{-}\right\rangle \simeq 1258$ [15]. At RHIC, at $\sqrt{s}_{N N}=200 \mathrm{GeV}$ in full phase space $\left\langle\pi^{+}+\pi^{-}\right\rangle \simeq 3343$ [16] leading to $V \approx 10^{4} \mathrm{fm}^{3}$. This extrapolation assumes very little variation of temperature and baryonchemical potentials from SPS to RHIC, which approximately holds [9]. In order to extrapolate from RHIC to LHC, we pragmatically use the saturation model (which proved to be successful in extrapolating multiplicity from SPS to RHIC) which predicts an increase in $\left\langle n_{\mathrm{ch}}\right\rangle$ by a factor $\simeq 4.5$ from $\sqrt{s}_{N N}=200 \mathrm{GeV}$ to $5.5 \mathrm{TeV}$ [17. This means that $V_{L H C} \approx 4.510^{4} \mathrm{fm}^{3}$ with a fair uncertainty up to a factor 2. By using the above values for $V$, and, conservatively, the upper estimates for $\left\langle\nu_{c}\right\rangle$ and $\left\langle\nu_{b}\right\rangle$ we obtain, from Eqs. (9) and (11) $R_{c}=\mathcal{O}\left(10^{-2}\right), R_{b}=\mathcal{O}\left(10^{-7}\right), R_{c b}=\mathcal{O}\left(10^{-4}\right)$ at RHIC and $R_{c}=\mathcal{O}(1), R_{b}=\mathcal{O}\left(10^{-4}\right), R_{c b}=\mathcal{O}\left(10^{-1}\right)$ at LHC, by using as input parameters $m_{c}, m_{b}, m_{u, d}$ and $T$ the same quoted below Eq. (91). Therefore, both at RHIC and LHC energies the formula (13) should be fairly accurate, because either the $R$ 's are $\ll 1$ or, like in the charm sector at LHC, $\left\langle\nu_{c}\right\rangle \gg 1$ and $R_{c} /\left\langle\nu_{c}\right\rangle \ll 1$.

We can now perform our predictions. To estimate the $\eta_{f}$ 's, we use the approximation $a_{f 1} \simeq g_{\mathrm{eff}} V\left[\left(m_{f}+\right.\right.$ $\left.\left.m_{u}\right) T / 2 \pi\right]^{3 / 2} \exp \left[\left(-m_{f}+m_{u}\right) / T\right]$ with input parameters like for the ratios $R_{f}$. We then obtain $\eta_{c} \approx 1.7-10$ and $\eta_{b} \approx(3.5-8.2) \cdot 10^{6}$ at RHIC and $\eta_{c} \approx 12-34$ and $\eta_{b} \approx(0.63-1.9) \cdot 10^{8}$ at LHC. With these numbers, assuming the mass of multiply heavy flavoured hadrons 
to be the sum of its quarks constituent masses, using Eq. (13) with $\lambda_{j}=1$ (i.e. taking vanishing chemical potentials) and appropriate spins, we get average primary yields of doubly charmed baryons (like $\Xi_{c c}$ and $\Omega_{c c}$ ) between $0.7 \cdot 10^{-4}$ and $7 \cdot 10^{-3}$ in central collisions at RHIC and between 0.019 and 0.38 at LHC. For mixed charmedbeautiful hadrons (like $\Xi_{b c}, \Omega_{b c}$ and $B_{c}$ meson), the yields should range between $4 \cdot 10^{-7}$ and $6 \cdot 10^{-5}$ at RHIC and $3 \cdot 10^{-4}$ and 0.022 at LHC. For doubly beautiful baryons (like $\Xi_{b b}$ and $\Omega_{b b}$ ), our estimates range between $2 \cdot 10^{-9}$ and $3 \cdot 10^{-8}$ at RHIC and between $2.6 \cdot 10^{-6}$ and $7 \cdot 10^{-5}$ at LHC. For the $\Omega_{c c c}$ baryon, the predicted yields are affected by a large uncertainty due to the cubic dependence on $\eta_{c}$; they range between $7 \cdot 10^{-7}$ and $10^{-4}$ at RHIC and between $10^{-3}$ and 0.03 at LHC. For charmed baryon yields at LHC, the predictions of the Eq. (13) turn out to be in good agreement with a preliminary calculation with the exact formula. All of the previous yields are enhanced by the feeding from heavier states, even by factor of about $4-5$; another factor $\approx 2$ comes from antiparticle yields. These factors should roughly compensate for the limited rapidity window accessible to experiments. Therefore, while at RHIC only doubly charmed hadrons seem to be within reach, at LHC, with a statistics of $10^{7}$ central events/year, doubly and triply charmed, charmed-beautiful, and perhaps doubly beautiful hadrons could in principle be observed.

We can now compare the above yields with the predictions by production models based on QCD hard scattering. At LHC, a model where heavy diquarks produced in $\mathcal{O}\left(\alpha_{S}^{4}\right)$ diagrams are assumed to fully hadronize into ccqbaryons, yields an upper bound on inclusive production of $\left(10^{-4}-10^{-3}\right)\left\langle\nu_{c}\right\rangle$ at $\sqrt{s}=14 \mathrm{TeV}$ [18] in pp, to be compared with $g_{\text {eff }}(0.8-2) \cdot 10^{-3}\left\langle\nu_{c}\right\rangle$ from coalescence in $\mathrm{HIC}$ at $\sqrt{s}_{N N}=5.5 \mathrm{TeV}$. A larger difference is found in the $\Xi_{b c}$ sector, where in p $\overline{\mathrm{p}}$ at $\sqrt{s}=1.8 \mathrm{TeV}$ the $1 \mathrm{~S}$ wave cross-section is predicted to be around $1 \mathrm{nb}$ [19], implying a ratio $\left\langle\Xi_{b c}\right\rangle /\left\langle\nu_{b}\right\rangle \sim 10^{-5}$ to be compared with $g_{\mathrm{eff}}^{1 S}(3-9) \cdot 10^{-4}$ from coalescence, i.e. at least one order of magnitude larger. Since the production process is $\mathcal{O}\left(\alpha_{S}^{6}\right)$, the difference is even larger for $\Omega_{c c c}$, for which a recent calculation [20] predicts a ratio $\left\langle\Omega_{c c c}\right\rangle /\left\langle\nu_{c}\right\rangle=\mathcal{O}\left(10^{-7}\right)$ in pp at $\sqrt{s}=14 \mathrm{TeV}$; this is between 2-3 orders of magnitude lower than our estimated ratio from coalescence at $\sqrt{s}_{N N}=5.5 \mathrm{TeV}$ i.e. $(0.1-1) \cdot 10^{-4}$. For charmonia and $\mathrm{B}_{c}$, the two mechanisms give closer predictions. For $\langle J / \psi\rangle /\left\langle\nu_{c}\right\rangle$, the difference is estimated to be a factor about 2.5 at LHC [12], whilst for $\mathrm{B}_{c}$ cross sections calculations 21] at $\sqrt{s}=14 \mathrm{TeV}$ imply a ratio $\left\langle\mathrm{B}_{c}\right\rangle /\left\langle\nu_{b}\right\rangle=\mathcal{O}\left(10^{-3}\right)$, around the same as from coalescence at $\sqrt{s}_{N N}=5.5 \mathrm{TeV}$. Also, it should be pointed out that, unlike $J / \psi$ and $\mathrm{B}_{c}$, multiply heavy flavoured hadrons have not been measured in hadronic collisions, so the predictions of the models based on hard scattering are still to be checked.

The predominant uncertainty on the previous estimates is that on heavy quark cross section. Other relevant uncertainties are the those on masses, effective degeneracy, extrapolated temperature and charged multiplicities, a modulation of the production as a function of rapidity as well as the replacement of the approximated formula (13) with the exact one. Yet, all these effects, which will be discussed in detail in a forthcoming paper [10], cannot alter the ratios of multiply to singly flavoured hadron yields by one order of magnitude. So the conclusion remains that if statistical coalescence scheme applies, a large enhancement in the measurement of $\left\langle\Xi_{b c}\right\rangle /\langle B\rangle$, which becomes dramatic for $\left\langle\Omega_{c c c}\right\rangle /\langle D\rangle$, could be found in heavy ion collisions with respect to pp at the LHC energy. This could be a clear indication of QGP formation.

\section{References}

[1] H. van Hees and R. Rapp, Phys. Rev. C 71, 034907 (2005).

[2] M. Schroedter, R. Thews and J. Rafelski, Phys. Rev. C 62, 024905 (2000)

[3] P. Braun-Munzinger and J. Stachel, Phys. Lett. B 490, 196 (2000).

[4] P. Levai, J. Zimany, Phys. Lett. B 304, 203 (1993).

[5] J. Schaffner-Bielich and A. Vischer, Phys. Rev. D 57, 4142 (1998).

[6] M. Gazdzicki and M. Gorenstein, Phys. Rev. Lett. 83, 4009 (1999).

[7] R. Thews, M. Schroedter and J. Rafelski, Phys. Rev. C 63, 054905 (2001); M. Gorenstein et al., Phys. Lett. B 509, 277 (2001); L. Grandchamp and R. Rapp, Phys. Lett. B 523, 60 (2001); A. Andronic et al., Phys. Lett.
B 571, 36 (2003); A. Kostyuk, nucl-th/0502005

[8] W. Broniowski and W. Florkowski, hep-ph/0202059

[9] F. Becattini et al., Phys. Rev. C 69, 024905 (2004)

[10] F. Becattini, A. Bieniek, in preparation.

[11] M. Cacciari, P. Nason and R. Vogt, hep-ph/0502203

[12] M. Bedjidian et al., hep-ph/0311048

[13] D. Miskowiec, http://www-linux.gsi.de/ misko/overlap/

[14] D. Kharzeev and K. Tuchin, Nucl. Phys. A 735, 248 (2004)

[15] S. Afanasiev et al., Phys. Rev. C 66, 054902 (2002)

[16] I. Bearden et al., nucl-ex/0403050

[17] M. Nardi, J. Phys. Conf. 5, 148 (2005)

[18] A. Berezhnoi et al., Phys. Rev. D 57, 4385 (1998)

[19] V. Kiselev and A. Likhoded, Phys. Usp. 45, 455 (2002)

[20] M. Nobary and R. Sepahvand, Phys. Rev. D 71, 034024 (2005)

[21] I. Gouz et al., Phys. Atom. Nucl. 67, 1559 (2004) 